\title{
Proceeding
}

Supplementary Issue: Spring Conferences of Sports Science. Costa Blanca Sports Science Events, 19-20 June 2020. Alicante, Spain.

\section{Who practices sports can be vegetarian?}

\author{
STEFANIA D'ANGELO $\triangle$, POMPILIO CUSANO
}

Department of Movement Sciences and Wellbeing, University of Naples "Parthenope", Naples, Italy

\begin{abstract}
In recent years, interest in "vegetarianism" has increased considerably, both among the common population and among sportsmen. Athletic performance and post-workout recovery can be improved through optimal nutrition: choosing the right foods and introducing them at the right time can make a difference in terms of sports results, for the same number of sessions and training hours. But those who practice sports can follow a vegetarian diet? The choice to become vegetarian is often based on the mere refusal to consume meat or animal products, a choice attributable to philosophical motivations of different nature. In this manuscript, we will try to determine, by commenting on the scientific data published in recent years, the effects, positive or negative, that could be a consequence from adopting a diet of this type, both in everyday life and in sports. Keywords: Athletes; Vegetarian; Nutrition; Diet; Sports.

Cite this article as:

D'Angelo, S. \& Cusano, P. (2020). Who practices sports can be vegetarian?. Journal of Human Sport and Exercise, 15(3proc), S552-S561. doi:https://doi.org/10.14198/jhse.2020.15.Proc3.08
\end{abstract}

\footnotetext{
Corresponding author. Department of Movement Sciences and Wellbeing, University of Naples "Parthenope", Naples, Italy. E-mail: stefania.dangelo@uniparthenope.it Supplementary Issue: Spring Conferences of Sports Science. Costa Blanca Sports Science Events, 19-20 June 2020. Alicante, Spain. JOURNAL OF HUMAN SPORT \& EXERCISE ISSN 1988-5202

(c) Faculty of Education. University of Alicante doi:10.14198/jhse.2020.15.Proc3.08
} 


\section{INTRODUCTION}

Athletes have many strategies they can use when fueling for performance. Nutrition can play a crucial role in optimizing training sessions as well as with recovery and metabolic adaptation. Sports medicine providers should help athletes navigate the many facets of sports nutrition, including food composition, nutrient timing, supplement use, and energy balance. Athletes should be able to obtain both adequate macronutrients (protein, carbohydrates, and fats) and micronutrients through a variety of foods. Additionally, athletes' nutritional requirements may vary widely depending on sport, position, timing of season, and training vs rest day. Most athletes will plan to either gain lean muscle mass, lose fat, or maintain their current body composition while not impeding their performance on the field. Athletes also need to be educated on the detrimental effects of rapid weight loss strategies for competitive advantage, especially in sports such as wrestling and gymnastics. Detrimental effects include hypo hydration and loss of glycogen stores and/or lean muscle mass (Bytomski, 2018). Athletes stress many of the metabolic pathways during training and may have increased micronutrient (vitamin and mineral) requirements. Most athletes should be able to obtain sufficient micronutrients through a well-balanced diet, but athletes who practice extreme dietary or weightloss patterns or eliminate whole food groups may be at risk for certain deficiencies (Bytomski, 2018; Motti et al, 2018; D'Angelo \& Tafuri, 2020).

Vegetarian diets have been repeatedly and consistently associated with improved health outcomes, as well as increased life expectancy, although the mechanism by which vegetarian diets improve health outcomes is not completely understood. Advocates of vegetarian diets often contend that vegetarian diets are more healthful, but the diet quality of vegetarian diets has not been systematically evaluated, making it difficult to exclude competing explanations such as increased health consciousness among vegetarians.

\section{BRIEF HISTORY OF VEGETARIAN NUTRITION}

In the last decades of the 20th century, plant-based nutrition became a topic of formal scientific inquiry. The attempt to identify the most beneficial diets for humans was initiated by ecologic data from the then science of epidemiology obtained by studies in African, Asian, and Mediterranean countries. These data showed that populations living in these regions had, on average, the lowest rates of diet-related no communicable diseases and lived a rather long life. Traditional Asian and Mediterranean diets are largely based on plant foods, suggesting that a plant-based diet provides health benefits beyond supplying nutrients in adequate amounts (Leitzmann, 2014).

The anatomic and physiologic characteristics of carnivores and herbivores in the animal kingdom compared with the characteristics of humans show that humans are omnivores by nature. However, plant foods may be the most important food source for human health. In former times, plants and their fruit were most likely always available, and they were easy to gather. Hunting animals was difficult and often dangerous, so that animal products were probably only consumed sporadically, except for short periods such as in the Paleolithic Era. It is likely that the consumption of small and slow-moving animals, eggs, and fish, may have played a role in the development of the human brain. With the introduction of agriculture, human diets became increasingly plant-centered (Leitzmann, 2014). The data on the early phase of vegetarian nutrition are fragmentary and are not always consistent. Some helpful information can be derived from the writings of historical authors. It is fairly clear that major early human cultures practiced a predominantly plant-based nutrition, but the precise historical extent of vegetarianism remains unknown (Craddock et al, 2016). 
Today, vegetarian nutrition has a growing international following. The increase in the number of vegetarians and vegans is attributable to health concerns and is also attributable to ethical, environmental, and social concerns. Vegetarians still remain a small minority in all countries except for India, where approximately onethird of the population is vegetarian. The data show that $\sim 10 \%$ of vegetarians are vegans (Leitzmann, 2014). The vegetarian movement is now supported by many leaders in the arts, sciences, music, and sports. In addition to the growing number of vegetarians and vegans, increasingly more people in Western countries continue to reduce their meat consumption. This can be documented by the average amount of meat consumed in a country such as Germany, where the consumption of meat has decreased by nearly $10 \%$ in the past $30 \mathrm{y}$. By comparison, meat consumption is still increasing in the United States and in most emerging economies.

\section{VEGETARIAN NUTRITION AND SPORT}

Though lacking a standardized definition, "vegetarian" generally describes a lacto-ovo vegetarian dietary pattern (herein referred to as vegetarian), which is free of meat, poultry, and fish; however, "vegetarian" is occasionally used interchangeably to describe more and less restrictive dietary patterns such as vegan (additionally eliminates eggs and dairy), semi-vegetarian (varying definitions), and pesco-vegetarian (consumes fish but not meat) (Rogerson, 2017) (Table 1). Eliminating meat, a defining attribute of vegetarian diets, is commonly presumed to contribute to improved health outcomes because higher consumption of cured, smoked, salted, or otherwise processed meat and/or red meat is consistently associated with increased risk of obesity, diabetes, heart disease, and mortality. Vegetarian diets can be rich in protective foods like vegetables, fruits, plant proteins, and whole grains that may improve health outcomes if consumed in place of meat. However, any healthful dietary pattern (e.g., Mediterranean Diet) can also be rich in these protective foods, and less healthful foods can also replace meat in vegetarian diets, underscoring the importance of assessing the diet quality of dietary patterns presumed to be more healthful (Parker \& Vadiveloo, 2019).

Table 1. Types of vegetarian diets.

\begin{tabular}{ll}
\hline Type & Description* \\
\hline Semi-vegetarian or & Occasionally consumes animal flesh (meat, poultry) and fish, eggs, dairy \\
Flexitarian & Excludes meat and poultry; includes fish, diary and eggs \\
Pescatarian & Excludes meat, fish and poultry; includes diary and eggs \\
Lacto-ovo-vegetarian & Excluded all flesh and eggs; includes dairy only \\
Lacto Vegetarian & Excluded all flesh and dairy; includes eggs only \\
Ovo Vegetarian & Excludes meat, fish, poultry, eggs and dairy \\
Vegan & Variable dietary restrictions; includes wild meat/game and fish in some \\
Macrobiotic vegetarian & variations of the diet \\
Fruitarian & Includes fruits, nuts, seeds and a some vegetables \\
\hline \multicolumn{2}{c}{ Note: ${ }^{*}$ (Rogerson, 2017) }
\end{tabular}

Athletes choose to follow specific dietary regimes to meet health and weight goals, reduce gastrointestinal distress, and ultimately improve sports performance, and/or for ethical or financial reasons. The prevalence of vegetarianism in athletes has only been examined in one study in 2014. Based on data collected at the Delhi 2010 Commonwealth Games, approximately one-third of all athletes $(n=351$ ) reported being vegetarian or meat avoiders. Those athletes most likely to adhere to a vegetarian or vegan diet included nonWestern athletes (prevalence $=31 \%$ ), those participating in a weight category sport (prevalence $=42 \%$ ), and 
female athletes (prevalence not reported) (Pelly \& Burkhart, 2014). Vegetarian athletes were associated with avoiding foods that contained additives and wheat, and cited nutrient composition as the main reason for following a vegetarian diet. Athletes can successfully follow a vegetarian diet with carefully planning to ensure adequate intake of energy, carbohydrate, protein, and nutrients (Cialdella-Kam et al., 2016).

\section{BENEFITS}

An individual's decision to change to a vegetarian way of life affects the quality of his or her nutritional status. The extent of nutrient deficiencies is higher in persons who become vegetarians for intellectual (ethical, moral, religious, spiritual) and social (ecologic, economic, political) reasons compared with those who turn to vegetarianism for physical (health, hygiene, toxicology, physical performance) reasons. The latter are primarily concerned about their health, and they wish to obtain information as to how to properly prepare vegetarian meals (Meyer \& Reguant-Closa, 2017).

Food is best eaten in its natural form or minimally processed. Nutrient losses by food processing, such as milling and heating, are considerable and are usually unknown to consumers. For example, nutrients lost by producing white flour from grain are immense. Bread, cakes, and pasta made from whole grains reduce blood cholesterol concentrations and decrease the risk of cancers of the digestive tract. This effect is mainly attributable to the higher dietary fiber content of whole-grain foods. However, isolated dietary fibers added to meals do not have the same effect.

Raw foods do not lose any nutrients by processing and need to be chewed thoroughly to procure the full benefit of their inherent nutritional value. Chewing also stimulates salivation and cleanses the teeth. Raw food increases satiety, supports digestion, and normalizes gut transit time. Last, but not least, raw food prevents overeating. The recommendations for raw food intake vary widely and range from almost no raw food (Ayurveda) to $100 \%$ raw food (strict raw food eaters). The German Nutrition Society recommends an intake of $100 \mathrm{~g} \mathrm{raw}$ food/d. The concept of wholesome nutrition recommends that approximately half the food consumed should be raw. It is worth noting that raw food may not always provide full health benefits. For example, certain phytochemicals (eg, $\beta$-carotene, lycopene) are absorbed to a much larger extent from cooked vegetables than from raw vegetables.

Some of the uncertainties voiced by vegetarians are related to the widely differing recommendations for critical nutrients across countries. Critical nutrients are those that are most often identified as not being supplied in sufficient amounts in certain population subgroups. Unexpectedly, there are only small differences in what constitutes a critical nutrient between meat eaters and vegetarians because most critical nutrients are the same for both groups. One exception is folate, which is considered a critical nutrient for meat eaters but presents no problem for vegetarians and vegans. In addition, vitamin B12 and possibly the omega-3 fatty acids are critical for vegans and for some, but not all, meat eaters or lacto-ovo-vegetarians (Leitzmann 2014).

\section{ANTIOXIDANT AND BENEFITS FOR ATHLETES}

Vegetarian diets have been associated with lower body mass index (BMI) and decreased risk of chronic disease (i.e., hypertension, type II diabetes, metabolic syndrome, and some cancers). However, exercise independently has also been associated with the same health benefits. To our knowledge, the impacts of potential synergistic or additive benefits of following both a physically active lifestyle and a vegetarian diet have not been examined. A well-planned vegetarian diet does include a high consumption of fruits and 
vegetables that provide dietary fiber and anti-inflammatory and immune-supporting micronutrients and phytochemicals.

Although oxidative stress has been observed during ageing, under certain pathological conditions (Ingrosso et al, 1995; Ingrosso et al, 1996; D'Angelo et al, 2012; D'Angelo et al, 2013), a number of studies have revealed that it is also related to contractile activity (D'Angelo \& Rosa, 2020). During exercise, oxygen consumption in muscle can increase 15-fold, leading to production of free radicals (or reactive oxygen species) that overwhelm the antioxidant protection system. High concentration of these species can be harmful to many cellular tissues, although some reactive molecules such as hydrogen peroxide and nitric oxide may serve functions in cellular signaling and as secondary messengers in moderate concentrations. High levels of reactive oxygen species (ROS) produced in skeletal muscle during exercise have been associated with muscle damage and impaired muscle function. Supporting endogenous defense systems with additional oral doses of antioxidants has received much attention as a noninvasive strategy to prevent or reduce oxidative stress, decrease muscle damage and improve exercise performance (Peternelj and Coombes, 2011).

Athletes should avoid foods that may increase oxidative stress and increase foods that are higher in antioxidants such as vitamins $\mathrm{C}$ and $\mathrm{E}$. Examples would be the Mediterranean diet (D'Angelo \& Cusano, 2020), which has a favorable effect on blood lipids and also protects against oxidative stress, and a diet high in fruits and vegetables, which raises plasma levels of antioxidants (D'Angelo, 2020) and protects against many chronic diseases. Vitamin $\mathrm{C}$ promotes collagen synthesis, facilitates glycogen storage, and may prevent exercise-induced oxidative changes. Vitamin $\mathrm{E}$ prohibits propagation of free radical formation. There is little evidence that antioxidant supplementation enhances athletic performance, although it may be beneficial in athletes recovering from injury. At high doses vitamins $C$ and $E$ can be pro-oxidant, and large doses of vitamin $\mathrm{E}$ can also impede vitamin K metabolism and platelet function (Bytomski, 2018; D'Angelo \& Rosa, 2020a; Meccariello et al, 2020).

\section{NUTRIENTS AND POSSIBLE DEFICIENCIES}

Athletes should be evaluated on an individual basis if there is concern for possible deficiency based on symptoms or performance decline. Hence, there are no general micronutrient guidelines for athletes. Common deficiencies include iron, vitamin $\mathrm{D}$, calcium, and some antioxidants such as vitamins $\mathrm{E}$ and $\mathrm{C}$. Vegetarians may require vitamin B12, iron, calcium, vitamin D, riboflavin, and zinc supplementation based on their food preferences. It is important to note that micronutrients are important for optimum health but are not considered to have ergogenic properties (Bytomski, 2018).

Any diet that restricts certain foods or food groups can increase the risk of low energy available and deficiencies in key nutrients such as protein, carbohydrates, essential fatty acids, and micronutrients (i.e., Bvitamins, calcium, vitamin $\mathrm{D}$, and iron). The athlete consuming a vegetarian diet often consumes more fruits and vegetables than the non-vegetarian athlete adding many nutritional benefits (i.e., providing phytochemicals and antioxidants to support exercise training and health.-Such diets would provide significant amounts of phytochemicals bioactive components, with nutraceutical (D'Angelo et al, 2019) effects due in part to their anti-oxidant (Zappia et al, 2010; D'Angelo \& Sammartino 2015; del Monaco et al, 2015; Vuoso et al, 2020) and anti-proliferative (D'Angelo et al, 2012a; D'Angelo et al, 2017; D'Angelo et al, 2019a; Martino et al, 2019; Boccellino et al, 2020) proprieties. 
One key nutritional concern is the high fiber content of the vegetarian diet, which may lead to early satiety and appetite blunting, reducing energy intake and contributing to low energy available. Vitamin $D$ is a concern for athletes who live in latitudes $>40^{\circ} \mathrm{N}$ as many athletes fail to meet recommendations from diet alone. In a systemic review and meta-analysis, Farrokhyar et al. (Farrokhyar et al, 2015) reported a high prevalence $(\sim 66 \%)$ of vitamin $\mathrm{D}$ deficiency among athletes, particularly in those who participated in indoor sports (i.e., swimming, basketball, dancing, gymnastics, volleyball, and wrestling). Good dietary sources of vitamin D include fatty fish and fortified products such as dairy products, cereals, and orange juice. Thus, athletes who do not consume dairy products and/or fish may be at increased risk for vitamin $D$ deficiency. For these individuals, a biochemical assessment of vitamin D status is warranted (Rogerson, 2017).

A vegetarian diet can provide adequate protein to support exercise training if the athlete has adequate energy intake. Thus, the concerns for nutrient deficiencies for vegetarian athletes are similar to those discussed for athletes with low energy availability (i.e., carbohydrates, protein, essential fatty acids, and certain micronutrients). Sports performance, however, has not been evaluated in vegetarian athletes (Cialdella-Kam et al, 2016).

The role of protein in the athlete's diet has garnered much attention over the years, and there has been ongoing debate about whether athletes require greater amounts of protein than non-athletic population. The consensus appears to be that athletes require more protein than the lay population. Data also indicates that protein requirements should be tailored to reflect sport-specific and training-goal requirements. Typical recommendations therefore include $1.6-1.7 \mathrm{~g} \cdot \mathrm{kg}^{-1} \cdot$ day $^{-1}$ for strength and power athletes and $1.2-1.4 \mathrm{~g}$. $\mathrm{kg}^{-1} \cdot$ day $^{-1}$ for endurance-sport athletes-values notably larger than the $0.8 \mathrm{~g} \cdot \mathrm{kg}^{-1} \cdot$ day $^{-1}$ recommended for most non-active adults. Values as high as $4.4 \mathrm{~g} \cdot \mathrm{kg}^{-1} \cdot$ day-1 have also been investigated in the literature recently, with favorable body-composition effects noted as a results of its composition (Lynch et al, 2018). Several features differentiate plant-based protein from animal-based protein, in addition to the source. Of the indispensable amino acids, branched-chain amino acids (BCAAs) are particularly important for promoting muscle protein synthesis (MPS) and include leucine, isoleucine, and valine. These amino acids are more concentrated in animal-based protein compared to plant protein (van Vliet et al, 2015). Digestion and absorption rates of different proteins can also differ and thus impact postprandial MPS rates. As such, some proteins (such as whey) are considered "fast" since they are rapidly digested, resulting in amino acids appearing quickly in the bloodstream. Other proteins, such casein and soy, protein are considered "slow" since they result in a slower, more prolonged, rate of absorption.

Carbohydrates are the main energy source during high-intensity activity for the central nervous system as well as muscular work. Carbohydrates are not all equal, as there is a vast difference between eating a bowl of oatmeal versus a bowl of ice cream. Whole grains, fruits, vegetables, and legumes are highly nutritious foods that are rich in antioxidants, fiber, vitamins, and minerals, while processed sugars abundant in the Western diet can be quite detrimental to health. Athletes have varied carbohydrate requirements based on training intensity, type of workout, and timing during their season. In general, athletes will need to consume 3 to $5 \mathrm{~g}$ per kilogram body weight daily for light activity and upward of 8 to $12 \mathrm{~g}$ per kilogram body weight per day for intense training.

Fat requirements for athletes are similar to those for non-athletes (20\%-35\% total daily calories should come from healthy fats). Athletes should focus on good sources of fat that are high in unsaturated fats and essential fatty acids. Trans fats should be avoided, and saturated fat should be less than $10 \%$ of total consumption. Healthy sources of fat include salmon, nuts and nut butters, and avocado, as well as coconut, olive oil, 
sesame oil, and canola oil. Athletes may also consider taking omega-3 supplements as they can also counteract inflammatory and free radical formation sustained from training (Bytomski, 2018).

\section{WHO PRACTICES SPORTS CAN BE VEGETARIAN?}

Yes, of course. Vegetarian diets are fully compatible with a very intense sporting activity, and are able to meet the needs of competitive athletes, by virtue of the high intake of carbohydrates which is fundamental for the athlete and more easily obtainable with this type of diet.

Vegetarian diets, thanks to their high content of carbohydrates and low in fat, are configured as optimal diets for those who play sports and are also able to meet the needs of competitive athletes. Vegetarian diets that meet energy needs and contain a variety of foods rich in plant protein guarantee an adequate amount of protein without requiring the use of special foods or supplements. Vegetarians, and especially vegans, still need to pay more attention than non-vegetarians in meeting energy needs due to the lower calorie content of their diet. Vegetarian athletes may have lower creatine muscle concentrations as this compound is mainly present in mammalian muscle tissue, therefore vegetarian athletes engaged in short duration and high intensity exercises or resistance training can benefit from creatine supplementation.

Interest and appreciation for plant-based diets continues to grow around the world, as both government agencies and various health and nutrition organizations promote the regular use of plant foods. The wide market offer of $100 \%$ vegetable foods facilitates adherence to this type of diet. Properly planned vegetarian diets provide an adequate supply of nutrients at all stages of the life cycle, and can also be useful in the therapeutic management of some chronic diseases. The overall nutritional composition, as assessed by the Alternative Healthy Eating Index, is generally better in vegetarian and vegan diets than in omnivorous diets. Although some vegetarian diets may be low in certain nutrients, such as calcium and vitamin B12, this aspect can be improved through appropriate planning.

Vegetarians are able to meet an increased protein requirement simply by increasing the calories consumed: lacto-ovo-vegetarians get on average $12.5 \%$ of the calories from proteins and vegans $11 \%$, which means that an $80 \mathrm{~kg}$ athlete who consumes $3,600 \mathrm{Kcal}$ per day obtains $1.41 \mathrm{~g}$ of protein per $\mathrm{kg}$ of body weight from a lacto-ovo-vegetarian diet and $1.2 \mathrm{~g}$ of protein per $\mathrm{kg}$ of body weight from a vegan diet. Increasing, even significantly, these values in a vegetarian diet by keeping the introduced calories constant is however feasible simply by increasing the quantity of legumes and decreasing the quantity of fruit and fats in meals.

As for iron, it must be said that especially women who practice intense sports are at risk of deficiency of this mineral (for any type of diet) and that low levels of ferritin in the blood can compromise athletic performance. Sportsmen should pay particular attention to the blood values of ferritin and, only in the case of a real deficiency, resort to supplements.

Studies carried out on vegetarian athletes show that their physical parameters have nothing to envy to those of omnivores, and a famous early-century study finds a significant prevalence of the physical performance of vegetarians over omnivores.

\section{CONCLUSION}

Sports nutrition is a key component for optimizing training, performance, injury prevention, and injury recovery. Nutritional recommendations need to be individualized for each athlete; thus, clinicians should be 
well informed to help determine proper caloric, macronutrient, micronutrient, and hydration intake based on the athlete's sport demands and training goals. An athlete's RMR along with his or her weight goals (loss, maintenance, or gain) are used to determine training macronutrient levels for protein, carbohydrate, and fat ratios. Micronutrient intake or supplementation should be determined based on identified nutrient deficiencies and/or dietary restrictions (eg, vegan, vegetarian) to assist with injury prevention and enhance injury recovery. Educating athletes on how to effectively fuel and hydrate for training sessions and competition based on the concept of nutrient timing is important to maximize performance. A comprehensive understanding of sports nutrition is crucial for clinicians to provide individualized and optimized recommendations (Bytomski, 2018).

Depending upon food preferences, eating patterns and exercise intensity, however, the diet of some athletes may contain suboptimal amounts of certain key nutrients, including total energy, protein, omega-3 fatty acids, calcium, vitamin D, iron, zinc, iodine, riboflavin and vitamin B12. In such cases, athletes can generally improve nutrient status through careful selection of foods containing the nutrient(s) they lack and a supplemental source when appropriate. Although research strongly suggests that a plant-based diet may offer many health benefits to athletes and nonathletic alike, there is currently little evidence that vegetarian diets per se are better than omnivorous diets for improving athletic training and performance.

However, the best confirmation of the validity of vegetarian diets for athletes is perhaps the fact that athletes such as Martina Navratilova, Carl Lewis (Olympic champion of long jump and vegan speed), Piero Venturato (twice world champion of all categories of bodybuilding, as well as seven times Italian champion and five times European champion), Edwin Moses (Olympic champion $400 \mathrm{~m}$ ), Paavo Nurmi (twenty times world champion of road walking), Emanuela Di Centa (Olympic cross-country champion), Alex Rabassa (32000 km of walking in about 500 days) and many others, are vegetarians.

\section{REFERENCES}

Boccellino M., Quagliuolo L., \& D'Angelo S. (2020). Annurca Apple Biophenols' Effects in Combination with Cisplatin on A549 Cells. Current Nutrition \& Food Science, 16, in press. https://doi.org/10.2174/1573401316999200504093028

Bytomski, J.R. (2018). Fueling for Performance. Sports health, 10, 1, 47-53. https://doi.org/10.1177/1941738117743913

Cialdella-Kam, L., Kulpins, D., \& Manore, M.M. (2016). Vegetarian, Gluten-Free, and Energy Restricted Diets in Female Athletes. Sports (Basel, Switzerland), 4(4), 50. https://doi.org/10.3390/sports4040050

Craddock, J.C., Probst, Y.C., \& Peoples, G.E. (2016). Vegetarian and Omnivorous Nutrition - Comparing Physical Performance. International journal of sport nutrition and exercise metabolism. 26(3), 212220. https://doi.org/10.1123/ijsnem.2015-0231

D'Angelo, S., Lembo, S., Flora, F., De Bonis, M.L., Balato, A., Ayala, F., Balato, N., Galletti, P., \& Zappia, V. (2012). Abnormal isoaspartyl residues in erythrocyte membranes from psoriatic patients. Archives of Dermatological Research, 304(6), 475-479. https://doi.org/10.1007/s00403-012-1247-z

D'Angelo S, La Porta R, Napolitano M, Galletti P, Quagliuolo L, \& Boccellino M.R. (2012)a. Effect of Annurca apple polyphenols on human HaCaT keratinocytes proliferation. Journal of Medicinal Food, 15(11), 1024-1031. https://doi.org/10.1089/jmf.2012.0076

D'Angelo, S., Trojsi, F., Salvatore, A., Daniele, L., Raimo, M., Galletti, P., \& Monsurrò, M.R. (2013). Accumulation of altered aspartyl residues in erythrocyte membrane proteins from patients with sporadic amyotrophic lateral sclerosis. Neurochemistry International, 63 (6), 626-34. https://doi.org/10.1016/..neuint.2013.09.006 
D'Angelo, S., Martino, E., llisso, C.P., Bagarolo, M.L., Porcelli, M. \& Cacciapuoti, G. (2017). Pro-oxidant and pro-apoptotic activity of polyphenol extract from Annurca apple and its underlying mechanisms in human breast cancer cells. International Journal of Oncology, 51, 939-948. https://doi.org/10.3892/ijo.2017.4088

D'Angelo S, \& Sammartino D. (2015). Protective effect of Annurca apple extract against oxidative damage in human erythrocytes. Current Nutrition \& Food Science, 11 (4), 248-256. https://doi.org/10.2174/1573401311666150610210529

D'Angelo, S., Martino E., \& Cacciapuoti, G. (2019)a. Effects of Annurca Apple (Malus pumila cv Annurca) Polyphenols on Breast Cancer Cells. Current Nutrition \& Food Science, 15 (7), 745-751. https://doi.org/10.2174/1573401315666190206142025

D'Angelo, S., Scafuro, M., \& Meccariello, R. (2019). BPA and Nutraceuticals, Simultaneous Effects on Endocrine Functions. Endocrine, Metabolic \& Immune Disorders - Drug Targets, 19(5), 594-604. https://doi.org/10.2174/1871530319666190101120119

D'Angelo S. \& Cusano P. (2020). Adherence to the Mediterranean diet in athletes. Sport Science, 13 (Suppl 1), 58-63.

D'Angelo S. \& Rosa R. (2020). Oxidative stress and sport performance. Sport Science, 13 (Suppl 1), 1822.

D'Angelo S. \& Rosa R. (2020) a. The impact of supplementation with Pomegranate fruit (Punica Granatum L.) on sport performance. Sport Science, 13 (Suppl 1), 29-37.

D'Angelo S. \& Tafuri D. (2020). Nutraceutical: their role in improving sports performance. Sport Science, 13 (Suppl 1): 7-12.

D'Angelo S. (2020). Polyphenols: Potential beneficial effects of these phytochemicals in athletes. Current Sports Medicine Reports. 19, 7, 260-265. https://doi.org/10.1249/jsr.0000000000000729

del Monaco, G., Officioso, A., D’Angelo, S., La Cara, F., lonata, E., Marcolongo, L., Squillaci, G., Maurelli, L., \& Morana, A. (2015). Characterization of extra virgin olive oils produced with typical Italian varieties by their phenolic profile. Food Chemistry, 184, 220-228. https://doi.org/10.1016/j.foodchem.2015.03.071

Ingrosso, D., D’Angelo, S., Perna, A.F., Iolascon, A., Miraglia del Giudice, E. Perrotta, S., Zappia, V., \& Galletti P. (1995). Increased membrane-protein methylation in hereditary spherocytosis. A marker of cytoskeletal disarray. European Journal of Biochemistry (The FEBS Journal), 228, 894-898. https://doi.org/10.1111/j.1432-1033.1995.0894m.x

Ingrosso, D., D'Angelo, S., Perrotta, S., d'Urzo, G., Iolascon, A., Perna, A.F., Galletti, P., Zappia, V. \& Miraglia del Giudice, E. (1996). Cytoskeletal behaviour in Spectrin and Band 3 deficient spherocytic red cells: evidence for a differentiated splenic conditioning role. British Journal of Haematology, 93, 38-41. https://doi.org/10.1046/j.1365-2141.1996.451990.x

Farrokhyar F., Tabasinejad R., Dao D., Peterson D., Ayeni O.R., Hadioonzadeh R., Bhandari M. (2015). Prevalence of vitamin D inadequacy in athletes: A systematic-review and meta-analysis. Sports Med, 45, 365-378. https://doi.org/10.1007/s40279-014-0267-6

Leitzmann, C. (2014). Vegetarian nutrition: past, present, future. The American Journal of Clinical Nutrition, 100 (suppl 1), 496S-502S. https://doi.org/10.3945/ajcn.113.071365

Lynch, H., Johnston, C., \& Wharton, C. (2018). Plant-Based Diets: Considerations for Environmental Impact, Protein Quality, and Exercise Performance. Nutrients, 10(12), 1841. https://doi.org/10.3390/nu10121841

Martino, E., Vuoso, D.C., D'Angelo, S., Mele, L., D'Onofrio, N., Porcelli, M., \& Cacciapuoti, G. (2019). Annurca apple polyphenol extract selectively kills MDA-MB-231 cells through ROS generation, sustained JNK activation and cell growth and survival inhibition. Scientific Reports, 10 (1), 13045. https://doi.org/10.1038/s41598-019-49631-x 
Meccariello, R., Santoro, A., D'Angelo, S., Morrone, R., Fasano, S., Viggiano, A., \& Pierantoni, R. (2020). The Epigenetics of the Endocannabinoid System. International Journal of Molecular Sciences, 21(3), 1113. https://doi.org/10.3390/ijms21031113

Meyer, N., \& Reguant-Closa, A. (2017). "Eat as If You Could Save the Planet and Win!" Sustainability Integration into Nutrition for Exercise and Sport. Nutrients, 9(4), 412. https://doi.org/10.3390/nu9040412

Motti, M.L., D’Angelo, S., \& Meccariello, R. (2018). MicroRNAs, Cancer and Diet: Facts and New Exciting Perspectives. Current Molecular Pharmacology, 11, 90-96. https://doi.org/10.2174/1874467210666171013123733

Parker, H.W., \& Vadiveloo, M.K. (2019). Diet quality of vegetarian diets compared with nonvegetarian diets: a systematic review. Nutrition reviews, 77(3), 144-160. https://doi.org/10.1093/nutrit/nuy067

Pelly F.E., \& Burkhart S.J. (2014). Dietary regimens of athletes competing at the delhi 2010 commonwealth games. International Journal of Sport Nutrition and Exercise Metabolism, 24, 28-36. https://doi.org/10.1123/ijsnem.2013-0023

Peternelj, T.T., \& Coombes, J.S. (2011). Antioxidant supplementation during exercise training: beneficial or detrimental? Sports medicine (Auckland, N.Z.), 41(12), 1043-1069. https://doi.org/10.2165/11594400-000000000-00000

Rogerson D. (2017). Vegan diets: practical advice for athletes and exercisers. Journal of the International Society of Sports Nutrition, 14, 36. https://doi.org/10.1186/s12970-017-0192-9

van Vliet, S., Burd, N.A., \& van Loon, L.J. (2015). The skeletal muscle anabolic response to plant-versus animal-based protein consumption. Journal of Nutrition, 145; 1981-1991. https://doi.org/10.3945/in.114.204305

Vuoso D.C., Porcelli M., Cacciapuoti G., \& D'Angelo S. (2020). Biological activity of MelAnnurca flesh apple biophenols. Current Nutrition \& Food Science. 16, in press. https://doi.org/10.2174/1573401316666200217113808

Zappia, V., Galletti, P., Manna, C., D’Angelo, S., Napoli, D., De Bonis, M.L., \& Capasso, G. (2010). Effects of Hydroxytyrosol on Cyclosporine Nephrotoxicity. In: Victor R. Preedy and Ronald Ross Watson, editors, Olives and Olive Oil in Health and Disease Prevention. Oxford: Academic Press, (pp. 1245-1252). https://doi.org/10.1016/b978-0-12-374420-3.00136-4

\section{(C) $(1) \Theta$}

This work is licensed under a Attribution-NonCommercial-NoDerivatives 4.0 International (CC BY-NC-ND 4.0). 